\title{
The Political Science 40o: With Citation Counts by Cohort, Gender, and Subfield
}

Hannah June Kim, University of California, Irvine

Bernard Grofman, University of California, Irvine

ABSTRACT This article updates the Masuoka, Grofman, and Feld 2002 dataset that identified the then-3,719 faculty in political science PhD-granting departments in the United States. That dataset contained information about each faculty member, including date and $\mathrm{PhD}$-granting department, lifetime citation counts, fields of interest, and school of employment. We similarly create a database with the 4,089 currently tenured or tenuretrack faculty, along with emeritus faculty, at US PhD-granting departments ca. 2017-2018. Using Google Scholar Profiles, along with manual counts for those who do not have a profile, we sort the dataset by citation count, PhD cohort, field of interest, and gender. This article identifies the 100 currently most-cited scholars, the 25 most-cited in each $\mathrm{PhD}$ cohort and subfield, the 40 most-cited women scholars, and the 25 most-cited emeriti. The full list of The Political Science 400 is available in an online appendix.

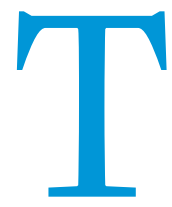

here are various ways to measure a scholar's professional visibility: (1) asking faculty to rank the scholars in their field(s) of expertise; (2) counting a faculty member's cumulative (or recent) publications, or perhaps only those in especially high-prestige journals, or weighting publications by the impact factor of the journal; and (3) counting cumulative (or recent) citations. We used the third approach. In this article, we duplicate the Kim-Grofman (KG) database with more recent data to replicate and extend the findings and rankings in "The Political Science 400: A 20-Year Update" by Masuoka, Grofman, and Feld (2007a), henceforth MGF. As in MGF and previous works (Klingemann 1986; Klingemann, Grofman, and Campagna 1989), we generated cumulative citation counts of all tenure-track political science faculty at the 133 $\mathrm{PhD}$-granting political science departments in the United States as of summer 2017. In the process of collecting information on currently employed faculty, we also gathered information on emeritus faculty. This resulted in a dataset of 4,089 faculty, of whom 3,412 are currently employed in tenured or tenure-track positions. For some analyses, the $\mathrm{N}$ is 3,769 because we are missing the $\mathrm{PhD}$ date for 320 scholars.

Although MGF and most other previous studies base citation counts on the Web of Science Social Science Citation Index

Hannah June Kim iD a PhD candidate in political science at the University of California, Irvine. She can be reached at hannah.kim@uci.edu.

Bernard Grofman iD is the Jack W. Peltason (Bren Foundation Endowed) Chair of Democracy Studies and professor of political science at the University of California, Irvine. He can be reached at bgrofman@uci.edu.
(SSCI), ${ }^{1}$ we opted to base ours on Google Scholar, which identifies citations to books as well as journal articles and other types of material. Our citation data from Google Scholar spans more than 50 years. In addition to Google Scholar citation count and university of present (or past) employment for each faculty member, we collected information on the $\mathrm{PhD}$ date, the institution from which the PhD was awarded, and the primary field of interest. We added information to the data fields collected by MGF on present rank and we also coded for gender. We provide information primarily in terms of lifetime citation counts because this best reflects overall visibility. This number obviously is biased in favor of those who have had more time to accumulate citations; therefore, similar to MGF, we also provide citation counts categorized separately by age cohort defined by $\mathrm{PhD}$ date, as well as by subfield and gender. However, in general, we report counts and other information separately for emeriti, focusing on those who are still professionally active, which we define as those who have published within the past five years.

Additional details about our methodology and general features of the KG dataset are in an online appendix.

\section{THE POLITICAL SCIENCE 400}

This update of The Political Science 400 again identifies the 400 most highly cited scholars in the profession who are currently teaching at PhD-granting departments in the United States, with their primary appointment in that department, by tallying the citations to lifetime bodies of work in all journals and books. It includes citation data from when scholars began receiving citations to their most recent work. The full list of The Political 
Science 400 is available in an online appendix, and the full dataset is available from the authors on request. Similar to MGF, for space reasons, the tables in this article limit the number of faculty displayed (e.g., table 1 lists only the 100 most-cited faculty). To further control for age effects similar to MGF, table 2 identifies the top 25 scholars from each five-year cohort. We also provide a list of the 40 most-cited women scholars with information on their PhD date, as well as the top 25 scholars in each subfield.

For the 100 most-cited scholars, table 1 identifies their PhD date, present affiliation, and where they received their $\mathrm{PhD}$, as well as their Google Scholar lifetime counts. We restrict table 1 to non-emeriti faculty so as to reflect the current state of the discipline. However, table 5 is a separate tabulation of the 25 most highly cited emeriti who are still professionally active.

Table 2 lists the 25 most-cited scholars from each five-year cohort. For younger cohorts, this may include some scholars who are not in the overall list of 400 ; for older cohorts, it may exclude some scholars who are in the overall list. As in table 1, table 2 also identifies their $\mathrm{PhD}$ date, present affiliation, and where they received their $\mathrm{PhD}$. For the three oldest cohorts, table 2 lists only those with more than 5,000 citations. We omit the data on post2015 PhDs because it is too soon for it to be representative.

Reviewing the full Political Science 400 dataset, we found that the plurality cohort is now $1975^{-1979}$, as compared to the plurality cohort of 1970-1974 in the MGF study. By examining the data in table 2, we also compared across cohorts the citation counts of the most-cited faculty within each cohort. which a citation from (and to) other subfields is common. Nonetheless, we can create an index of overrepresentation by dividing each subfield's share of The Political Science 400 by its share of faculty in the full dataset. Although that table is omitted for space reasons, we note that overrepresentation is highest in methodology (i.e., a ratio value of 1.90) and underrepresentation is greatest in political theory (i.e., a ratio value of 0.54 ).

Table 4 lists the 40 most-cited women scholars. As in table 3, table 4 also identifies their $\mathrm{PhD}$ date, present affiliation, and where they received their $\mathrm{PhD}$. All 40 women are in The Political Science 400.

We also found that females comprise a very low proportion of older cohorts but a more substantial proportion of the youngest four cohorts among The Political Science 40o. We also note that there are more women in The Political Science 400 in the subfields of comparative politics, American politics, and international relations relative to the overall proportion of women in those subfields. They are significantly underrepresented in the subfields of methodology and political theory relative to the overall proportion of women in those subfields. (These tables were omitted due to space limitations.)

\section{EMERITI ONLY}

If they were included, emeriti would comprise $17.3 \%$ of The Political Science 400, displacing some non-emeritus faculty. Therefore, we chose not to include them in table 1 . However, we also thought it a mistake to disregard them because these faculty are some of

\section{Our main goal was to update the MGF (2007a) dataset with data from more than a decade later and to switch from SSCI data to Google Scholar data, which includes more complete information about book citations.}

Table 3 lists the 25 most-cited scholars from each field. ${ }^{2}$ As in table 2, table 3 also identifies their PhD date, present affiliation, and where they received their $\mathrm{PhD}$.

Not all subfields are represented equally in The Political Science 40o. Some subfields contain more faculty than others; however, subfields also differ in their citation practices regarding the mean number of citations per publication and in the extent to the most highly visible in the discipline. Moreover, as expected, the most highly cited faculty in this group remain professionally active (i.e., publishing during the past five years, 2013-2018). All but five of the 100 most-cited emeriti are still professionally active.

Table 5 lists the 25 most-cited professionally active emeritus scholars. If not for their emeritus status, 69 active emeriti would

Table 1

Top 100 Most-Cited Scholars

\begin{tabular}{|c|c|c|c|c|c|}
\hline First Name & Last Name & 2017 University & PhD University & Year & Count \\
\hline Ronald & Inglehart & U of Michigan & $\mathrm{U}$ of Chicago & 1967 & 94,125 \\
\hline Robert O. & Keohane & Stanford U & Harvard U & 1966 & 89,856 \\
\hline Robert & Axelrod & U of Michigan & Yale U & 1969 & 71,958 \\
\hline Nancy & Fraser & New School for Social Research & SUNY & 1980 & 63,820 \\
\hline Gary & King & Harvard U & U of Wisconsin-Madison & 1984 & 62,048 \\
\hline Barry R. & Weingast & Stanford U & Caltech & 1978 & 57,747 \\
\hline Jon & Elster & Columbia U & $\mathrm{U}$ of Paris & 1972 & 54,727 \\
\hline James & Scott & Yale U & Yale U & 1967 & 54,367 \\
\hline
\end{tabular}


Table 1 (Continued)

First Name

Theda

Adam

Peter A.

B. Guy

Paul

Jon A.

Larry

Stephen D.

Seyla

Todd

Andrew

James D.

Shanto

Mathew D.

Gary

Peter J.

Morris $\mathrm{P}$.

Donald

James $\mathrm{H}$.

David

John

Donald

Stephan

Brian J. L.

Kathleen

Henry E.

W. Lance

Mark J.

Robert

Alfred

Gary W.

David D.

Liesbet

Terry M.

Susan

Kenneth

Kay Lehman

Scott

Howard

Herbert P.

George

Frank R.

James

Kenneth J.

John

Bryan
Last Name

Skocpol

Przeworski

Hall

Peters

Pierson

Krosnick

Diamond

Krasner

Benhabib

Sandler

Moravcsik

Fearon

lyengar

McCubbins

Marks

Katzenstein

Fiorina

Green

Fowler

Sears

Mearsheimer

Kinder

Haggard

Berry

Thelen

Brady

Bennett

Miller

Jervis

Stepan

Cox

Laitin

Hooghe

Moe

Rose-Ackerman

Shepsle

Schlozman

Mainwaring

Rosenthal

Kitschelt

Tsebelis

Baumgartner

Thurber

Meier

Zaller

Jones
2017 University

Harvard U

NYU

Harvard U

$\mathrm{U}$ of Pittsburgh

UC Berkeley

Stanford U

Stanford U

Stanford U

Yale U

$\mathrm{U}$ of Texas, Dallas

Princeton U

Stanford U

Stanford U

Duke U

UNC Chapel Hill

Cornell U

Stanford U

Columbia U

UC San Diego

UCLA

$\mathrm{U}$ of Chicago

$U$ of Michigan

UC San Diego

U of Texas, Dallas

MIT

UC Berkeley

$\mathrm{U}$ of Washington

$U$ of Delaware

Columbia U

Columbia U

Stanford U

Stanford U

UNC Chapel Hill

Stanford U

Yale U

Harvard U

Boston College

Harvard U

NYU

Duke U

$U$ of Michigan

UNC Chapel Hill

American U

Texas A\&M

UCLA

UT Austin
PhD University

Harvard U

Northwestern U

Harvard U

Michigan State U

Yale U

$U$ of Michigan

Stanford U

Harvard U

Yale U

SUNY Binghamton

Harvard U

UC Berkeley

$U$ of lowa

Caltech

Stanford U

Princeton U

$U$ of Rochester

UC Berkeley

Harvard U

Yale U

Cornell U

UCLA

UC Berkeley

$\mathrm{U}$ of Washington

UC Berkeley

MIT

Yale U

U of Wisconsin-Madison

UC Berkeley

Columbia U

Caltech

UC Berkeley

U of Leuven

$\mathrm{U}$ of Minnesota

Yale $U$

$\mathrm{U}$ of Rochester

$U$ of Chicago

Stanford U

MIT

Bielefeld U

Washington U, St. Louis

$\mathrm{U}$ of Michigan

Indiana U

Syracuse U

UC Berkeley

UT Austin

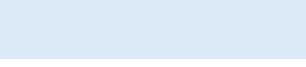


Table 1 (Continued)

\begin{tabular}{|c|c|c|c|c|c|}
\hline First Name & Last Name & 2017 University & PhD University & Year & Count \\
\hline Michael & Laver & NYU & U of Liverpool & 1981 & 20,438 \\
\hline Amy & Gutmann & U of Pennsylvania & Harvard U & 1976 & 19,876 \\
\hline Michael & Sandel & Harvard U & U of Oxford & 1981 & 19,871 \\
\hline Peter M. & Haas & U of Massachusetts, Amherst & MIT & 1986 & 19,749 \\
\hline Mahmood & Mamdani & Columbia U & Harvard U & 1974 & 19,507 \\
\hline Robert & Bates & Harvard U & MIT & 1969 & 19,482 \\
\hline Edward G. & Carmines & Indiana U & SUNY Buffalo & 1975 & 19,370 \\
\hline Nathaniel & Beck & NYU & Yale U & 1977 & 19,087 \\
\hline Matthew S. & Shugart & UC Davis & UC Irvine & 1988 & 18,891 \\
\hline David & Collier & UC Berkeley & U of Chicago & 1971 & 18,878 \\
\hline John & Aldrich & Duke U & U of Rochester & 1975 & 18,644 \\
\hline Bruce & Bueno de Mesquita & NYU & $\mathrm{U}$ of Michigan & 1971 & 18,614 \\
\hline John & Roemer & Yale U & UC Berkeley & 1974 & 18,550 \\
\hline John & Stephens & UNC Chapel Hill & Yale U & 1976 & 18,331 \\
\hline Michael & Scriven & Claremont Graduate U & Oxford U & 1956 & 18,142 \\
\hline James & Mahoney & Northwestern U & UC Berkeley & 1997 & 17,954 \\
\hline Steven & Levitsky & Harvard U & UC Berkeley & 1999 & 17,265 \\
\hline James $\mathrm{N}$. & Druckman & Northwestern U & UC San Diego & 1999 & 17,213 \\
\hline John & Gerring & UT Austin & UC Berkeley & 1993 & 17,188 \\
\hline Margaret & Levi & Stanford U & Harvard U & 1974 & 16,861 \\
\hline Keith T. & Poole & U of Georgia & U of Rochester & 1978 & 16,861 \\
\hline Bernard & Grofman & UC Irvine & U of Chicago & 1972 & 16,768 \\
\hline Helen & Milner & Princeton U & Harvard U & 1986 & 16,711 \\
\hline Wendy & Brown & UC Berkeley & Princeton U & 1983 & 16,416 \\
\hline Robert & Erikson & Columbia U & U of Illinois & 1969 & 16,383 \\
\hline Laurence J. & O'Toole & U of Georgia & Syracuse U & 1975 & 16,317 \\
\hline M. Kent & Jennings & UC Santa Barbara & U of North Carolina & 1961 & 16,265 \\
\hline Alfred & Blumstein & Carnegie Mellon U & Cornell U & 1961 & 16,135 \\
\hline Hank & Jenkins-Smith & U of Oklahoma & U of Rochester & 1985 & 16,071 \\
\hline Vivien A. & Schmidt & Boston U & U of Chicago & 1981 & 16,025 \\
\hline James & Snyder & Harvard U & Caltech & 1985 & 15,940 \\
\hline Stanley & Feldman & SUNY Stony Brook & U of Minnesota & 1978 & 15,848 \\
\hline G. Bingham & Powell & U of Rochester & Stanford U & 1968 & 15,825 \\
\hline William & Connolly & Johns Hopkins U & U of Michigan & 1977 & 15,812 \\
\hline Beth & Simmons & U of Pennsylvania & Harvard U & 1991 & 15,780 \\
\hline Timothy P. & Mitchell & Columbia U & Princeton U & 1984 & 15,757 \\
\hline Stephen & Ansolabehere & Harvard U & Harvard U & 1989 & 15,634 \\
\hline Frances Fox & Piven & CUNY Graduate School & U of Chicago & 1962 & 15,519 \\
\hline James & Stimson & UNC Chapel Hill & UNC Chapel Hill & 1970 & 15,518 \\
\hline Larry M. & Bartels & Vanderbilt U & UC Berkeley & 1983 & 15,507 \\
\hline Jack & Snyder & Columbia U & Columbia U & 1981 & 15,387 \\
\hline Paul M. & Sniderman & Stanford U & UC Berkeley & 1968 & 15,270 \\
\hline Robert & Shapiro & Columbia U & U of Chicago & 1982 & 15,207 \\
\hline G. John & Ikenberry & Princeton U & U of Chicago & 1985 & 15,135 \\
\hline Benjamin I. & Page & Northwestern U & Stanford U & 1973 & 15,014 \\
\hline Michael & Bratton & Michigan State U & Brandeis U & 1978 & 14,948 \\
\hline Michael & Hechter & Arizona State U & Columbia U & 1972 & 14,890 \\
\hline
\end{tabular}


Table 2

Most-Cited Top 25 Scholars in Each Cohort

\begin{tabular}{|c|c|c|c|c|}
\hline First Name & Last Name & 2017 University & PhD University & Count \\
\hline \multicolumn{5}{|c|}{ Cohort 1: 1950-1954 (N=1) } \\
\hline John & Brademas & NYU & Oxford U & 404 \\
\hline \multicolumn{5}{|c|}{ Cohort 2: 1955-1959 (N=2) } \\
\hline Brian J. L. & Berry & U of Texas, Dallas & U of Washington & 25,080 \\
\hline Peter & Woll & Brandeis U & Cornell U & 670 \\
\hline \multicolumn{5}{|c|}{ Cohort 3: 1960-1964 (N=20) } \\
\hline David & Sears & UCLA & Yale U & 26,167 \\
\hline Howard & Rosenthal & NYU & MIT & 21,458 \\
\hline M. Kent & Jennings & UC Santa Barbara & U of North Carolina & 16,265 \\
\hline Alfred & Blumstein & Carnegie Mellon U & Cornell U & 16,135 \\
\hline Frances Fox & Piven & CUNY Graduate School & U of Chicago & 15,519 \\
\hline Benjamin J. & Cohen & UC Santa Barbara & Columbia U & 9,857 \\
\hline Kenneth & Prewitt & Columbia U & Stanford U & 5,352 \\
\hline Harvey & Mansfield & Harvard U & Harvard U & 3,272 \\
\hline David B. & Lyons & Boston U & Harvard U & 3,207 \\
\hline Allan & Kornberg & Duke U & U of Michigan & 2,920 \\
\hline David & Holloway & Stanford U & Cambridge $U$ & 2,620 \\
\hline Claude E. & Welch Jr. & SUNY Buffalo & Oxford U & 2,500 \\
\hline Sheldon W. & Simon & Arizona State U & U of Minnesota & 1,593 \\
\hline Michael & Lofchie & UCLA & UC Berkeley & 1,546 \\
\hline James C. & Hsiung & NYU & Columbia U & 593 \\
\hline Charles & Parrish & Wayne State U & UNC Chapel Hill & 418 \\
\hline Robert K. & Faulkner & Boston College & U of Chicago & 200 \\
\hline Gilbert M. & Cuthbertson & Rice U & Harvard U & 74 \\
\hline $\mathrm{Jim}$ & Leach & U of lowa & Princeton U & 50 \\
\hline \multicolumn{5}{|c|}{ Cohort 4: 1965-1969 (N=73) } \\
\hline Ronald & Inglehart & U of Michigan & U of Chicago & 94,125 \\
\hline Robert O. & Keohane & Stanford U & Harvard U & 89,856 \\
\hline Robert & Axelrod & U of Michigan & Yale U & 71,958 \\
\hline James & Scott & Yale U & Yale U & 54,367 \\
\hline Adam & Przeworski & NYU & Northwestern U & 46,562 \\
\hline Robert & Jervis & Columbia U & UC Berkeley & 24,409 \\
\hline Alfred & Stepan & Columbia U & Columbia U & 24,384 \\
\hline Robert & Bates & Harvard U & MIT & 19,482 \\
\hline Robert & Erikson & Columbia U & U of Illinois & 16,383 \\
\hline G. Bingham & Powell & U of Rochester & Stanford U & 15,825 \\
\hline Paul M. & Sniderman & Stanford U & UC Berkeley & 15,270 \\
\hline Peter C. & Ordeshook & Caltech & U of Rochester & 14,601 \\
\hline Paul E. & Peterson & Harvard U & U of Chicago & 14,104 \\
\hline Edward D. & Mansfield & U of Pennsylvania & U of Pennsylvania & 13,925 \\
\hline Steven & Brams & NYU & Northwestern U & 13,890 \\
\hline Robert & Kaufman & Rutgers U & Harvard U & 10,614 \\
\hline James A. & Caporaso & U of Washington & U of Pennsylvania & 9,753 \\
\hline Milton & Lodge & SUNY Stony Brook & U of Michigan & 9,184 \\
\hline G. David & Garson & North Carolina State U & Harvard U & 9,174 \\
\hline Ira & Katznelson & Columbia U & Cambridge U & 8,540 \\
\hline
\end{tabular}


Table 2 (Continued)

First Name

Paul R.

Michael

John

Douglas

Thomas

Cohort

Jon

B. Guy

Stephen D.

Todd

Shanto

Morris $\mathrm{P}$.

W. Lance

David D.

Susan

Kenneth

Kay Lehman

James

Bryan

Mahmood

David

Bruce

John

Margaret

Bernard

James

Benjamin I.

Michael

Richard S

Eric M.

Susan

Barry R.

Theda

Seyla

Donald

Mark J.

Terry M.

Herbert P.

Kenneth J.

Amy

Edward G

Nathaniel

John

John

Keith T.

Laurence J.
Last Name

Abramson

Shapiro

Jackson

Rae

Schwartz

Elster

Peters

Krasner

Sandler

lyengar

Fiorina

Bennett

Laitin

Rose-Ackerman

Shepsle

Schlozman

Thurber

Jones

Mamdani

Collier

Bueno de Mesquita

Roemer

Levi

Grofman

Stimson

Page

Hechter

Katz

Uslaner

Welch

=193)

Weingast

Skocpol

Benhabib

Kinder

Miller

Moe

Kitschelt

Meier

Gutmann

Carmines

Beck

Aldrich

Stephens

Poole

O'Toole
2017 University

Michigan State U

U of Hawaii

$\mathrm{U}$ of Michigan

Yale U

UCLA

Columbia U

$U$ of Pittsburgh

Stanford U

U of Texas, Dallas

Stanford U

Stanford U

$\mathrm{U}$ of Washington

Stanford U

Yale U

Harvard U

Boston College

American U

UT Austin

Columbia U

UC Berkeley

NYU

Yale U

Stanford U

UC Irvine

UNC Chapel Hill

Northwestern U

Arizona State U

Johns Hopkins U

$U$ of Maryland

Pennsylvania State U

Stanford U

Harvard U

Yale U

$U$ of Michigan

$U$ of Delaware

Stanford U

Duke U

Texas A\&M

$U$ of Pennsylvania

Indiana U

NYU

Duke U

UNC Chapel Hill

$U$ of Georgia

U of Georgia
PhD University

Count

UC Berkeley $\quad 8,434$

Northwestern U

7,505

Harvard U

7,307

$U$ of Wisconsin

6,751

$\mathrm{U}$ of Pittsburgh

6,481

$\mathrm{U}$ of Paris

54,727

Michigan State $U$

42,666

Harvard U

32,851

SUNY Binghamton

31,849

$U$ of lowa

29,947

$U$ of Rochester

27,731

Yale U

24,731

UC Berkeley

23,819

Yale U

22,474

U of Rochester 22,248

U of Chicago 22,090

Indiana U

21,134

UT Austin

20,491

Harvard U

19,507

$\mathrm{U}$ of Chicago

18,878

$\mathrm{U}$ of Michigan

18,614

UC Berkeley

18,550

Harvard U

16,861

$\mathrm{U}$ of Chicago $\quad 16,768$

UNC Chapel Hill $\quad 15,518$

Stanford U 15,014

Columbia U $\quad 14,890$

Yale U 14,624

Indiana U $\quad 13,694$

U of Illinois, Urbana Champaign $\quad 13,547$

Caltech $\quad 57,747$

Harvard U $\quad 47,410$

Yale U 32,194

UCLA 25,922

U of Wisconsin-Madison $\quad 24,642$

$\mathrm{U}$ of Minnesota $\quad 22,508$

Bielefeld U $\quad 21,443$

Syracuse U $\quad 20,610$

Harvard U $\quad 19,876$

SUNY Buffalo $\quad 19,370$

Yale U 19,087

U of Rochester $\quad 18,644$

Yale U 18,331

U of Rochester $\quad 16,861$

Syracuse U $\quad 16,317$

(continued) 
Table 2 (Continued)

First Name

Stanley

William

Michael

Joshua

Michael

Evelyne

James L.

Hal G.

Robert

Richard

\section{Cohort 7: 1980-1984 (N=231)}

Nancy Fraser

Gary King

Peter A. Hal

Larry

Mathew D.

Gary

John

Stephan

Henry E.

Gary W.

Scott

John

Michael

Michael

Wendy

Vivien A.

Timothy P.

Larry M.

Jack

Robert

Jack A.

Lee

David A.

Kaare

Jack

Cohor

Paul

Jon A.

Donald

Kathleen

Liesbet

George

Frank R.

Peter M.

Matthew S.

Helen

Hank
Last Name

Feldman

Connolly

Bratton

Cohen

Doyle

Huber

Gibson

Rainey

Huckfeldt

Hall

Diamond

McCubbins

Marks

Mearsheimer

Haggard

Brady

Cox

Mainwaring

Zaller

Laver

Sandel

Brown

Schmidt

Mitchell

Bartels

Snyder

Shapiro

Goldstone

Epstein

Lake

Strom

Donnelly
2017 University

SUNY Stony Brook

Johns Hopkins U

Michigan State U

Stanford U

Columbia U

UNC Chapel Hill

Washington U

$U$ of Georgia

UC Davis

Rutgers U

New School for Social Research

Harvard U

Harvard U

Stanford U

Duke U

UNC Chapel Hill

$U$ of Chicago

UC San Diego

UC Berkeley

Stanford U

Harvard U

UCLA

NYU

Harvard U

UC Berkeley

Boston U

Columbia U

Vanderbilt U

Columbia U

Columbia U

George Mason U

Washington U

UC San Diego

UC San Diego

U of Denver

UC Berkeley

Stanford U

Columbia U

MIT

UNC Chapel Hill

$U$ of Michigan

UNC Chapel Hill

$\mathrm{U}$ of Massachusetts, Amherst

UC Davis

Princeton U

U of Oklahoma
PhD University

Count

U of Minnesota $\quad 15,848$

$\mathrm{U}$ of Michigan

15,812

Brandeis U

14,948

Harvard U

14,841

Harvard U

14,757

Yale U

14,326

U of lowa

14,264

Ohio State U

13,439

Washington U, St. Louis

13,321

UCLA

13,027

SUNY

63,820

U of Wisconsin-Madison $\quad 62,048$

Harvard U 43,305

Stanford U 33,133

Caltech 28,764

Caltech

Stanford U 28,594

Cornell U 26,092

UC Berkeley 25,166

MIT

24,855

Caltech 24,354

Stanford U 21,610

UC Berkeley $\quad 20,541$

U of Liverpool $\quad 20,438$

U of Oxford $\quad 19,871$

Princeton U $\quad 16,416$

U of Chicago $\quad 16,025$

Princeton U 15,757

UC Berkeley $\quad 15,507$

Columbia U $\quad 15,387$

U of Chicago $\quad 15,207$

Harvard U $\quad 14,549$

Emory U $\quad 14,365$

Cornell U $\quad 13,744$

Stanford U $\quad 13,667$

UC Berkeley $\quad 13,438$

Yale U $\quad 35,966$

U of Michigan $\quad 34,143$

UC Berkeley $\quad 27,208$

UC Berkeley $\quad 24,903$

24,903

U of Leuven $\quad 22,676$

Washington U, St. Louis $\quad 21,440$

U of Michigan $\quad 21,432$

MIT 19,749

UC Irvine $\quad 18,891$

Harvard U $\quad 16,711$

U of Rochester $\quad 16,071$

(continued) 
Table 2 (Continued)

First Name

James

Stephen

G. John

Sven

Diana

Scott L.

Jane

Susan

Jack

Barbara

Brendan

Norman

Shaun

Gary

Cohort 9: 1990-1994 (N=335)

\section{Andrew}

James D.

Peter J.

John

Beth

Jonathan $\mathrm{N}$

Jose A.

Andrew

Alan

Nicolas

Arthur

R. Michael

Stathis N.

Burt L.

Alastair lain

Bruce

Lawrence R.

Martin

John

Christopher

Kurt

Mark P.

Devesh

Wendy

John

\section{Cohort \\ 10: 1995-1999 ( $N=367)$}

James

Steven

James $\mathrm{N}$.

Michael L.

Nicholas

Robert B.

Torben
Last Name

Ansolabehere

Ikenberry

Steinmo

Mutz

Page

Bennett

Stokes

Knight

Geddes

O'Leary

Schofield

Bowler

Goertz

Moravcsik

Fearon

Katzenstein

Gerring

Simmons

Katz

Cheibub

Bennett

Gerber

van de Walle

Lupia

Alvarez

Kalyvas

Monroe

Johnston

Bimber

Jacobs

Gilens

Huber

Ansell

Weyland

Jones

Kapur

Rahn

Brehm

Mahoney

Levitsky

Druckman

Ross

Sambanis

Reich

Iversen
2017 University

Harvard U

Harvard U

Princeton U

$\mathrm{U}$ of Colorado

$\mathrm{U}$ of Pennsylvania

$U$ of Michigan

Johns Hopkins U

Yale U

Duke U

UCLA

$U$ of Pennsylvania

Washington $U$

UC Riverside

U of Notre Dame

Princeton U

Stanford U

Cornell U

UT Austin

$\mathrm{U}$ of Pennsylvania

Caltech

Texas A\&M

Georgetown U

Yale U

Cornell U

$\mathrm{U}$ of Michigan

Caltech

Yale U

Pennsylvania State U

Harvard U

UC Santa Barbara

$U$ of Minnesota

Princeton U

Columbia U

UC Berkeley

UT Austin

Rice $U$

U of Pennsylvania

$U$ of Minnesota

$U$ of Chicago

Northwestern U

Harvard U

Northwestern U

UCLA

$\mathrm{U}$ of Pennsylvania

Stanford U

Harvard U
PhD University

Count

Caltech

15,940

Harvard U

15,634

$\mathrm{U}$ of Chicago

15,135

UC Berkeley

14,146

Stanford U

12,154

$U$ of Michigan

11,541

$U$ of Massachusetts

10,612

Stanford U 10,154

U of Chicago $\quad 9,775$

UC Berkeley $\quad 9,467$

London School of Economics $\quad 9,372$

Essex U $\quad 8,939$

Washington U, St. Louis $\quad 8,930$

U of Michigan $\quad 8,923$

Harvard U $\quad 31,683$

UC Berkeley $\quad 31,287$

Princeton U 28,166

UC Berkeley $\quad 17,188$

Harvard U $\quad 15,780$

UC San Diego $\quad 13,712$

U of Chicago $\quad 13,416$

Harvard U $\quad 12,838$

MIT $\quad 12,648$

Princeton U 11,951

Caltech 11,317

Duke U 10,624

U of Chicago $\quad 10,536$

Oxford U 9,773

U of Michigan $\quad 9,104$

MIT $\quad 8,798$

Columbia U $\quad 8,762$

\begin{tabular}{rr}
8,762 \\
\hline$C$ Berkeley
\end{tabular}

C,390

U of Rochester $\quad 7,880$

$\mathrm{U}$ of Chicago $\quad 7,752$

Stanford U $\quad 7,566$

U of Michigan $\quad 7,487$

Princeton $U \quad 6,905$

U of Minnesota $\quad 6,899$

U of Michigan $\quad 6,773$

UC Berkeley $\quad 17,954$

UC Berkeley $\quad 17,265$

UC San Diego $\quad 17,213$

Princeton U 14,014

Princeton U $\quad 13,703$

Stanford U $\quad 13,499$

Duke $U \quad 13,328$

(continued) 
Table 2 (Continued)

\section{First Name}

Daniet

Daniel

Alastair

David

Mark

Cas

Clark C.

Aseem

Caroline J.

Mark M.

Matt

Leslie

Bernard

Daniel

James Raymond

Peter R.

Andrew D

Jasjeet S.

Nicholas

Cohort 11: 2000-2004 (N=440)

James $\mathrm{H}$

Latanya

Kosuke

Michael

Jacob

Michele

Macartan

Kenneth F.

Stuart

Jeremy M

Jonathan

Luke

Emilie

Anirudh

Gregory

William

David

Adam

Zachary

Bernard

Jennifer

Markus

Dustin

Jon C. W.

Aníbal

Coh

Mona

Idean
Last Name

Treisman

Smith

Lazer

Pollack

Mudde

Gibson

Prakash

Tolbert

Blyth

Golder

McCall

Manin

Posner

Vreeland

Andreas

Martin

Sekhon

Valentino

Fowler

Sweeney

Imai

Tomz

Hacker

Betsill

Humphreys

Scheve

Soroka

Weinstein

Rodden

Keele

Hafner-Burton

Krishna

Huber

Howell

Campbell

Berinsky

Elkins

Harcourt

Gandhi

Prior

Tingley

Pevehouse

Pérez-Liñán

Krook

Salehyan
2017 University

PhD University

Count

UCLA

NYU

Northeastern U

Temple U

$U$ of Georgia

UC San Diego

$\mathrm{U}$ of Washington

$U$ of lowa

Brown U

Pennsylvania State U

CUNY Graduate School

NYU

UCLA

Georgetown U

Brown U

$\mathrm{U}$ of Michigan

UC Berkeley

$U$ of Michigan

UC San Diego

Harvard U

Princeton U

Stanford U

Yale U

Colorado State U

Columbia U

Stanford U

$U$ of Michigan

Stanford U

Stanford U

Georgetown U

UC San Diego

Duke U

Yale U

$U$ of Chicago

U of Notre Dame

MIT

UT Austin

Columbia U

Emory U

Princeton U

Harvard U

$\mathrm{U}$ of Wisconsin-Madison

$\mathrm{U}$ of Pittsburgh

Stanford U

Rutgers $U$

Columbia U

7,294

5,868

5,076 
Table 2 (Continued)

First Name

Gabriel

Thad

Jason

David

Jeffrey

Daniel

Matthew

Erica

Jacob N.

Rocio

Dan

Joseph

Matt

Taylor

Milan

Daniel

Kyle

Boris

Matthew

Justin

Michael

Philipp

Cohort 13: 2010-2014 (N=501)

\section{Jennifer K.}

Suzanne

Andrew

Alison

Daniel

Daniel

Teppei

Matthew

Brian

Justin

Christopher J.

Margaret E.

Bethany

Simeon

Michael

Rikhil R.

Max

Ragnhild

Reed

Toby

Pablo

Noam

Adam

Allan

Paul
Last Name

Lenz

Dunning

Seawright

Nickerson

Church

Hopkins

Levendusky

Edwards

Shapiro

Titiunik

Slater

Wright

Barreto

Boas

Svolik

Aldrich

Beardsley

Shor

Kroenig

Phillips

Findley

Rehm

Lobasz

Mettler

Delton

Gash

Kinderman

Smith

Yamamoto

Blackwell

Min

Grimmer

Fariss

Roberts

Lacina

Nichter

Mille

Bhavnani

Abrahms

Nordas

Wood

Bolsen

Querubin

Lupu

Bonica

Dafoe

Staniland
2017 University

UC Berkeley

UC Berkeley

Northwestern U

Temple U

$\cup$ of Houston

U of Pennsylvania

$\mathrm{U}$ of Pennsylvania

Miami U

Princeton U

$\mathrm{U}$ of Michigan

$U$ of Chicago

Pennsylvania State U

UCLA

Boston U

Yale U

Northeastern U

Duke U

$\mathrm{U}$ of Houston

Georgetown U

Columbia U

UT Austin

Ohio State U

U of Delaware

Cornell U

SUNY Stony Brook

$U$ of Oregon

$U$ of Delaware

Harvard U

MIT

Harvard U

$\mathrm{U}$ of Michigan

Stanford U

$U$ of Michigan

UC San Diego

$U$ of Rochester

UC San Diego

George Washington U

$U$ of Wisconsin-Madison

Northeastern U

$U$ of Michigan

Arizona State U

Georgia State U

NYU

Vanderbilt U

Stanford U

Yale U

U of Chicago
PhD University

Count

Princeton U

4,390

UC Berkeley

4,273

UC Berkeley

3,823

Yale U

3,416

U of Notre Dame $\quad 3,263$

Harvard U $\quad 3,175$

Stanford U $\quad 3,100$

UNC Chapel Hill $\quad 2,640$

Stanford U 2,560

UC Berkeley $\quad 2,559$

Emory U 2,522

UCLA $\quad 2,520$

UC Irvine $\quad 2,488$

UC Berkeley $\quad 2,362$

U of Chicago $\quad 2,343$

Harvard U 2,313

UC San Diego $\quad 2,175$

Columbia U $\quad 2,150$

UC Berkeley $\quad 1,998$

UC San Diego $\quad 1,969$

$\mathrm{U}$ of Illinois $\quad 1,966$

Duke $U \quad 1,932$

Duke $U$

of Minnesota $\quad 5,029$

U of Chicago $\quad 3,189$

UC Santa Barbara $\quad 2,909$

UC Berkeley $\quad 2,768$

Cornell U 2,595

UC San Diego $\quad 2,505$

Princeton U 2,492

Harvard U $\quad 2,404$

UCLA $\quad 2,252$

Harvard U 2,143

UC San Diego $\quad 2,001$

Harvard U $\quad 1,973$

Stanford U $\quad 1,870$

UC Berkeley $\quad 1,793$

Princeton U $\quad 1,690$

$\begin{array}{ll}\text { Stanford } U & 1,687\end{array}$

UCLA $\quad 1,593$

Norwegian U of Science and Technology $\quad 1,371$

UNC Chapel Hill $\quad 1,212$

Northwestern U $\quad 1,210$

MIT $\quad 1,176$

Princeton U $\quad 1,173$

NYU $\quad 1,107$

UC Berkeley $\quad 1,089$

MIT

1,042 
Table 3

Most-Cited Top 25 Scholars from Each Field

\begin{tabular}{|c|c|c|c|c|c|}
\hline First Name & Last Name & 2017 University & PhD University & Year & Count \\
\hline \multicolumn{6}{|c|}{ American Politics ( $\mathrm{N}=1002)$} \\
\hline Barry R. & Weingast & Stanford U & Caltech & 1978 & 57,747 \\
\hline Paul & Pierson & UC Berkeley & Yale U & 1989 & 35,966 \\
\hline Jon A. & Krosnick & Stanford U & U of Michigan & 1986 & 34,143 \\
\hline Shanto & lyengar & Stanford U & U of lowa & 1973 & 29,947 \\
\hline Mathew D. & McCubbins & Duke U & Caltech & 1983 & 28,764 \\
\hline Morris P. & Fiorina & Stanford U & U of Rochester & 1972 & 27,731 \\
\hline Donald & Green & Columbia U & UC Berkeley & 1988 & 27,208 \\
\hline David & Sears & UCLA & Yale U & 1962 & 26,167 \\
\hline Donald & Kinder & $\mathrm{U}$ of Michigan & UCLA & 1975 & 25,922 \\
\hline Gary W. & Cox & Stanford U & Caltech & 1983 & 24,354 \\
\hline Terry M. & Moe & Stanford U & U of Minnesota & 1976 & 22,508 \\
\hline Kay Lehman & Schlozman & Boston College & U of Chicago & 1973 & 22,090 \\
\hline Howard & Rosenthal & NYU & MIT & 1964 & 21,458 \\
\hline Kenneth J. & Meier & Texas A\&M & Syracuse U & 1975 & 20,610 \\
\hline John & Zaller & UCLA & UC Berkeley & 1984 & 20,541 \\
\hline Bryan & Jones & UT Austin & UT Austin & 1970 & 20,491 \\
\hline Edward G. & Carmines & Indiana U & SUNY Buffalo & 1975 & 19,370 \\
\hline John & Aldrich & Duke U & U of Rochester & 1975 & 18,644 \\
\hline James N. & Druckman & Northwestern U & UC San Diego & 1999 & 17,213 \\
\hline Keith T. & Poole & U of Georgia & U of Rochester & 1978 & 16,861 \\
\hline Robert & Erikson & Columbia U & U of Illinois & 1969 & 16,383 \\
\hline William & Connolly & Johns Hopkins U & U of Michigan & 1977 & 15,812 \\
\hline Stephen & Ansolabehere & Harvard U & Harvard U & 1989 & 15,634 \\
\hline James & Stimson & UNC Chapel Hill & UNC Chapel Hill & 1970 & 15,518 \\
\hline Larry M. & Bartels & Vanderbilt U & UC Berkeley & 1983 & 15,507 \\
\hline \multicolumn{6}{|c|}{ Comparative Politics $(\mathrm{N}=827)$} \\
\hline Ronald & Inglehart & U of Michigan & U of Chicago & 1967 & 94,125 \\
\hline James & Scott & Yale U & Yale U & 1967 & 54,367 \\
\hline Theda & Skocpol & Harvard U & Harvard U & 1975 & 47,410 \\
\hline Adam & Przeworski & NYU & Northwestern U & 1966 & 46,562 \\
\hline Peter A. & Hall & Harvard U & Harvard U & 1982 & 43,305 \\
\hline Larry & Diamond & Stanford U & Stanford U & 1980 & 33,133 \\
\hline Gary & Marks & UNC Chapel Hill & Stanford U & 1982 & 28,594 \\
\hline Stephan & Haggard & UC San Diego & UC Berkeley & 1983 & 25,166 \\
\hline Kathleen & Thelen & MIT & UC Berkeley & 1987 & 24,903 \\
\hline W. Lance & Bennett & $\mathrm{U}$ of Washington & Yale U & 1974 & 24,731 \\
\hline Mark J. & Miller & U of Delaware & U of Wisconsin-Madison & 1978 & 24,642 \\
\hline Alfred & Stepan & Columbia U & Columbia U & 1969 & 24,384 \\
\hline David D. & Laitin & Stanford U & UC Berkeley & 1974 & 23,819 \\
\hline Liesbet & Hooghe & UNC Chapel Hill & $\mathrm{U}$ of Leuven & 1989 & 22,676 \\
\hline Herbert P. & Kitschelt & Duke U & Bielefeld U & 1979 & 21,443 \\
\hline George & Tsebelis & U of Michigan & Washington U St. Louis & 1985 & 21,440 \\
\hline Mahmood & Mamdani & Columbia U & Harvard U & 1974 & 19,507 \\
\hline Matthew S. & Shugart & UC Davis & UC Irvine & 1988 & 18,891 \\
\hline David & Collier & UC Berkeley & $\mathrm{U}$ of Chicago & 1971 & 18,878 \\
\hline John & Roemer & Yale U & UC Berkeley & 1974 & 18,550 \\
\hline John & Stephens & UNC Chapel Hill & Yale U & 1976 & 18,331 \\
\hline Steven & Levitsky & Harvard U & UC Berkeley & 1999 & 17,265 \\
\hline John & Gerring & UTAustin & UC Berkeley & 1993 & 17,188 \\
\hline
\end{tabular}


Table 3 (Continued)

\begin{tabular}{|c|c|c|c|c|c|}
\hline First Name & Last Name & 2017 University & PhD University & Year & Count \\
\hline Margaret & Levi & Stanford U & Harvard U & 1974 & 16,861 \\
\hline Helen & Milner & Princeton U & Harvard U & 1986 & 16,711 \\
\hline \multicolumn{6}{|c|}{ International Relations ( $\mathbf{N = 7 7 8 ) ~}$} \\
\hline Robert O. & Keohane & Stanford U & Harvard U & 1966 & 89,856 \\
\hline Robert & Axelrod & U of Michigan & Yale U & 1969 & 71,958 \\
\hline Stephen D. & Krasner & Stanford U & Harvard U & 1972 & 32,851 \\
\hline Seyla & Benhabib & Yale U & Yale U & 1977 & 32,194 \\
\hline Todd & Sandler & $\mathrm{U}$ of Texas Dallas & SUNY Binghamton & 1971 & 31,849 \\
\hline Andrew & Moravcsik & Princeton U & Harvard U & 1992 & 31,683 \\
\hline James D. & Fearon & Stanford U & UC Berkeley & 1992 & 31,287 \\
\hline Peter J. & Katzenstein & Cornell U & Princeton U & 1992 & 28,166 \\
\hline John & Mearsheimer & U of Chicago & Cornell U & 1980 & 26,092 \\
\hline Robert & Jervis & Columbia U & UC Berkeley & 1968 & 24,409 \\
\hline Susan & Rose-Ackerman & Yale U & Yale U & 1970 & 22,474 \\
\hline Scott & Mainwaring & Harvard U & Stanford U & 1983 & 21,610 \\
\hline Peter M. & Haas & U of Massachusetts Amherst & MIT & 1986 & 19,749 \\
\hline Robert & Bates & Harvard U & MIT & 1969 & 19,482 \\
\hline Bruce & Bueno de Mesquita & NYU & U of Michigan & 1971 & 18,614 \\
\hline Alfred & Blumstein & Carnegie Mellon U & Cornell U & 1961 & 16,135 \\
\hline Beth & Simmons & $\mathrm{U}$ of Pennsylvania & Harvard U & 1991 & 15,780 \\
\hline Jack & Snyder & Columbia U & Columbia U & 1981 & 15,387 \\
\hline G. John & Ikenberry & Princeton U & U of Chicago & 1985 & 15,135 \\
\hline Michael & Doyle & Columbia U & Harvard U & 1977 & 14,757 \\
\hline Edward D. & Mansfield & U of Pennsylvania & U of Pennsylvania & 1969 & 13,925 \\
\hline David A. & Lake & UC San Diego & Cornell U & 1984 & 13,744 \\
\hline Hal G. & Rainey & U of Georgia & Ohio State U & 1978 & 13,439 \\
\hline Jack & Donnelly & U of Denver & UC Berkeley & 1982 & 13,438 \\
\hline Torben & Iversen & Harvard U & Duke U & 1995 & 13,328 \\
\hline \multicolumn{6}{|c|}{ Methodology (N=129) } \\
\hline Gary & King & Harvard U & U of Wisconsin-Madison & 1984 & 62,048 \\
\hline Jon & Elster & Columbia U & U of Paris & 1972 & 54,727 \\
\hline James H. & Fowler & UC San Diego & Harvard U & 2003 & 26,956 \\
\hline Henry E. & Brady & UC Berkeley & MIT & 1980 & 24,855 \\
\hline Kenneth & Shepsle & Harvard U & U of Rochester & 1970 & 22,248 \\
\hline Michael & Laver & NYU & U of Liverpool & 1981 & 20,438 \\
\hline Nathaniel & Beck & NYU & Yale U & 1977 & 19,087 \\
\hline James & Mahoney & Northwestern U & UC Berkeley & 1997 & 17,954 \\
\hline Bernard & Grofman & UC Irvine & U of Chicago & 1972 & 16,768 \\
\hline M. Kent & Jennings & UC Santa Barbara & U of North Carolina & 1961 & 16,265 \\
\hline James & Snyder & Harvard U & Caltech & 1985 & 15,940 \\
\hline Peter C. & Ordeshook & Caltech & U of Rochester & 1969 & 14,601 \\
\hline Sven & Steinmo & U of Colorado & UC Berkeley & 1987 & 14,146 \\
\hline Latanya & Sweeney & Harvard U & MIT & 2001 & 12,359 \\
\hline Kosuke & Imai & Princeton U & Harvard U & 2003 & 11,564 \\
\hline Scott L. & Page & U of Michigan & U of Michigan & 1985 & 11,541 \\
\hline James E. & Alt & Harvard U & $U$ of Essex & 1978 & 9,000 \\
\hline Harold D. & Clarke & U of Texas Dallas & Duke U & 1971 & 8,727 \\
\hline Michael C. & Munger & Duke U & Washington U & 1984 & 7,417 \\
\hline Robert & Powell & UC Berkeley & UC Berkeley & 1985 & 7,266 \\
\hline Thomas & Romer & Princeton U & Yale U & 1974 & 7,115 \\
\hline Steven E. & Finkel & U of Pittsburgh & SUNY Stony Brook & 1984 & 7,057 \\
\hline
\end{tabular}


Table 3 (Continued)

\begin{tabular}{|c|c|c|c|c|c|}
\hline First Name & Last Name & 2017 University & PhD University & Year & Count \\
\hline Janet & Box-Steffensmeier & Ohio State U & $\mathrm{U}$ of Texas & 1993 & 6,751 \\
\hline Thomas & Schwartz & UCLA & $U$ of Pittsburgh & 1969 & 6,481 \\
\hline Jasjeet S. & Sekhon & UC Berkeley & Cornell U & 1999 & 6,257 \\
\hline \multicolumn{6}{|c|}{ Political Theory $(\mathrm{N}=346)$} \\
\hline Nancy & Fraser & New School for Social Research & SUNY & 1980 & 63,820 \\
\hline Amy & Gutmann & U of Pennsylvania & Harvard U & 1976 & 19,876 \\
\hline Michael & Sandel & Harvard U & U of Oxford & 1981 & 19,871 \\
\hline Wendy & Brown & UC Berkeley & Princeton U & 1983 & 16,416 \\
\hline Joshua & Cohen & Stanford U & Harvard U & 1979 & 14,841 \\
\hline Robert B. & Reich & Stanford U & Stanford U & 1998 & 13,499 \\
\hline Jean Louise & Cohen & Columbia U & New School for Social Research & 1979 & 12,417 \\
\hline Jane & Bennett & Johns Hopkins U & U of Massachusetts & 1986 & 10,612 \\
\hline Joan & Tronto & U of Minnesota & Princeton U & 1981 & 10,527 \\
\hline G David & Garson & North Carolina State U & Harvard U & 1969 & 9,174 \\
\hline Susan & Buck-Morss & Cornell U & Georgetown U & 1975 & 9,107 \\
\hline Charles & Beitz & Princeton U & Princeton U & 1978 & 8,871 \\
\hline Drucilla & Cornell & Rutgers U & UCLA & 1981 & 8,462 \\
\hline Michael & Shapiro & U of Hawaii & Northwestern U & 1966 & 7,505 \\
\hline Bernard & Manin & NYU & Institut d'Etudes Politiques & 1995 & 7,135 \\
\hline Josiah & Ober & Stanford U & U of Michigan & 1980 & 7,016 \\
\hline Alexander & Rosenberg & Duke U & John Hopkins U & 1971 & 5,968 \\
\hline Simone & Chambers & UC Irvine & Columbia U & 1989 & 5,680 \\
\hline Bernard & Harcourt & Columbia U & Harvard U & 2000 & 5,577 \\
\hline Stephen & Holmes & NYU & Yale U & 1976 & 5,317 \\
\hline Charles & Larmore & Brown U & Yale U & 1978 & 4,509 \\
\hline Russell L. & Hanson & Indiana U & U of Minnesota & 1982 & 4,356 \\
\hline James & Enelow & UT Austin & U of Rochester & 1977 & 4,168 \\
\hline Bertell & Ollman & NYU & Oxford U & 1967 & 3,856 \\
\hline Kathy & Ferguson & U of Hawaii & U of Minnesota & 1976 & 3,818 \\
\hline \multicolumn{6}{|c|}{ Public Policy, Public Administration, Public Law, Political Psychology ( $N=330)$} \\
\hline B. Guy & Peters & U of Pittsburgh & Michigan State U & 1970 & 42,666 \\
\hline Brian J. L. & Berry & U of Texas, Dallas & U of Washington & 1958 & 25,080 \\
\hline Frank R. & Baumgartner & UNC Chapel Hill & U of Michigan & 1986 & 21,432 \\
\hline James & Thurber & American U & Indiana U & 1974 & 21,134 \\
\hline Laurence J. & O'Toole & U of Georgia & Syracuse U & 1975 & 16,317 \\
\hline Hank & Jenkins-Smith & U of Oklahoma & U of Rochester & 1985 & 16,071 \\
\hline Stanley & Feldman & SUNY Stony Brook & U of Minnesota & 1978 & 15,848 \\
\hline Benjamin I. & Page & Northwestern U & Stanford U & 1973 & 15,014 \\
\hline Lee & Epstein & Washington U & Emory U & 1983 & 14,365 \\
\hline James L. & Gibson & Washington U & U of lowa & 1975 & 14,264 \\
\hline David & Lazer & Northeastern U & U of Michigan & 1996 & 11,811 \\
\hline William & Berry & Florida State U & U of Minnesota & 1980 & 10,974 \\
\hline James H. & Kuklinski & U of Illinois Urbana & U of Illinois, Urbana Champaign & 1975 & 10,215 \\
\hline H. Brinton & Milward & U of Arizona & Ohio State U & 1978 & 9,997 \\
\hline Milton & Lodge & SUNY Stony Brook & U of Michigan & 1967 & 9,184 \\
\hline Paul & Diehl & U of Texas, Dallas & U of Michigan & 1983 & 8,874 \\
\hline Gregory A. & Caldeira & Ohio State U & Princeton U & 1978 & 8,857 \\
\hline Leonie & Huddy & SUNY Stony Brook & UCLA & 1989 & 7,783 \\
\hline Leslie & McCall & CUNY Graduate School & U of Wisconsin-Madison & 1995 & 7,508 \\
\hline John & Mollenkopf & CUNY Graduate School & Harvard U & 1974 & 7,322 \\
\hline Pamela & Conover & UNC Chapel Hill & U of Minnesota & 1979 & 6,991 \\
\hline
\end{tabular}


Table 3 (Continued)

\begin{tabular}{|c|c|c|c|c|c|}
\hline First Name & Last Name & 2017 University & PhD University & Year & Count \\
\hline Wendy & Rahn & $U$ of Minnesota & U of Minnesota & 1990 & 6,899 \\
\hline Thomas H. & Jackson & U of Rochester & Yale U & 1975 & 6,849 \\
\hline John & Brehm & U of Chicago & U of Michigan & 1990 & 6,773 \\
\hline Edella & Schlager & U of Arizona & Indiana U & 1990 & 6,487 \\
\hline
\end{tabular}

Table 4

Top 40 Most-Cited Women Scholars (Excluding Emerita=1,135)

\begin{tabular}{|c|c|c|c|c|c|}
\hline First Name & Last Name & 2017 University & PhD University & Year & Count \\
\hline Nancy & Fraser & New School for Social Research & SUNY & 1980 & 63,820 \\
\hline Theda & Skocpol & Harvard U & Harvard U & 1975 & 47,410 \\
\hline Seyla & Benhabib & Yale U & Yale U & 1977 & 32,194 \\
\hline Kathleen & Thelen & MIT & UC Berkeley & 1987 & 24,903 \\
\hline Liesbet & Hooghe & UNC Chapel Hill & U of Leuven & 1989 & 22,676 \\
\hline Susan & Rose-Ackerman & Yale U & Yale U & 1970 & 22,474 \\
\hline Kay Lehman & Schlozman & Boston College & U of Chicago & 1973 & 22,090 \\
\hline Amy & Gutmann & U of Pennsylvania & Harvard U & 1976 & 19,876 \\
\hline Margaret & Levi & Stanford U & Harvard U & 1974 & 16,861 \\
\hline Helen & Milner & Princeton U & Harvard U & 1986 & 16,711 \\
\hline Wendy & Brown & UC Berkeley & Princeton U & 1983 & 16,416 \\
\hline Vivien $\mathrm{A}$. & Schmidt & Boston U & U of Chicago & 1981 & 16,025 \\
\hline Beth & Simmons & U of Pennsylvania & Harvard U & 1991 & 15,780 \\
\hline Frances Fox & Piven & CUNY Graduate School & U of Chicago & 1962 & 15,519 \\
\hline Lee & Epstein & Washington U & Emory U & 1983 & 14,365 \\
\hline Evelyne & Huber & UNC Chapel Hill & Yale U & 1977 & 14,326 \\
\hline Susan & Welch & Pennsylvania State U & U of Illinois, Urbana Champaign & 1970 & 13,547 \\
\hline Latanya & Sweeney & Harvard U & MIT & 2001 & 12,359 \\
\hline Diana & Mutz & U of Pennsylvania & Stanford U & 1988 & 12,154 \\
\hline Terry L. & Karl & Stanford U & Stanford U & 1982 & 11,193 \\
\hline Jane & Bennett & Johns Hopkins U & U of Massachusetts & 1986 & 10,612 \\
\hline Joan & Tronto & U of Minnesota & Princeton $\mathrm{U}$ & 1981 & 10,527 \\
\hline Susan & Stokes & Yale U & Stanford U & 1988 & 10,154 \\
\hline Judith L. & Goldstein & Stanford U & UCLA & 1983 & 9,852 \\
\hline Barbara & Geddes & UCLA & UC Berkeley & 1986 & 9,467 \\
\hline Susan & Buck-Morss & Cornell U & Georgetown U & 1975 & 9,107 \\
\hline Drucilla & Cornell & Rutgers U & UCLA & 1981 & 8,462 \\
\hline Holli A. & Semetko & Emory U & LSE & 1987 & 8,401 \\
\hline Caroline J. & Tolbert & U of lowa & U of Colorado, Boulder & 1996 & 8,217 \\
\hline Lisa & Martin & U of Wisconsin-Madison & Harvard U & 1989 & 8,148 \\
\hline Michele & Betsill & Colorado State U & U of Colorado & 2000 & 7,908 \\
\hline Leonie & Huddy & SUNY Stony Brook & UCLA & 1989 & 7,783 \\
\hline Deborah & Brautigam & Johns Hopkins U & Tufts U & 1987 & 7,712 \\
\hline Jean C. & Oi & Stanford U & U of Michigan & 1983 & 7,635 \\
\hline Elizabeth J. & Perry & Harvard U & U of Michigan & 1978 & 7,591 \\
\hline Leslie & McCall & CUNY Graduate School & U of Wisconsin-Madison & 1995 & 7,508 \\
\hline Valerie Jane & Bunce & Cornell U & U of Michigan & 1976 & 7,038 \\
\hline Pamela & Conover & UNC Chapel Hill & U of Minnesota & 1979 & 6,991 \\
\hline Virginia & Gray & U of Minnesota & Washington $U$ & 1972 & 6,921 \\
\hline Wendy & Rahn & U of Minnesota & U of Minnesota & 1990 & 6,899 \\
\hline
\end{tabular}


Table 5

Top 25 Most-Cited Still Active Emeritus Scholars (N=677)

\begin{tabular}{|c|c|c|c|c|c|}
\hline First Name & Last Name & 2017 University & PhD University & Year & Count \\
\hline Robert & Putnam & Harvard U & Yale U & 1970 & 175,198 \\
\hline Sidney & Verba & Harvard U & Princeton U & 1959 & 70,059 \\
\hline Arend & Lijphart & UC San Diego & Yale U & 1963 & 49,327 \\
\hline Philippe & Schmitter & Stanford U & UC Berkeley & 1967 & 46,129 \\
\hline Sidney & Tarrow & Cornell U & UC Berkeley & 1965 & 42,820 \\
\hline David & Soskice & Duke U & Oxford U & 1969 & 33,524 \\
\hline Bruce & Russett & Yale U & Yale U & 1961 & 27,820 \\
\hline Carole & Pateman & UCLA & Oxford U & 1971 & 25,702 \\
\hline John W. & Kingdon & U of Michigan & U of Wisconsin-Madison & 1965 & 23,924 \\
\hline Robert & Gilpin & Princeton U & UC Berkeley & 1960 & 21,978 \\
\hline Anne-Marie & Slaughter & Princeton U & Oxford U & 1966 & 21,629 \\
\hline John & Ferejohn & Stanford U & Stanford U & 1972 & 19,591 \\
\hline Irene S. & Rubin & Northern Illinois U & U of Chicago & 1977 & 19,229 \\
\hline Michael S. & Lewis-Beck & U of lowa & U of Michigan & 1973 & 19,213 \\
\hline Margaret & Keck & Johns Hopkins U & Columbia U & 1986 & 19,126 \\
\hline Richard G. & Niemi & U of Rochester & U of Michigan & 1967 & 18,948 \\
\hline William & Crotty & Northeastern U & UNC Chapel Hill & 1964 & 18,534 \\
\hline Edward & Tufte & Yale U & Yale U & 1968 & 18,451 \\
\hline Donald L. & Horowitz & Duke U & Harvard U & 1967 & 18,317 \\
\hline Wesley G. & Skogan & Northwestern U & Northwestern U & 1971 & 17,969 \\
\hline Robert A. & Kagan & UC Berkeley & Yale U & 1974 & 17,401 \\
\hline Ole R. & Holsti & Duke U & Stanford U & 1962 & 17,319 \\
\hline Gary A. & Orfield & UCLA & U of Chicago & 1968 & 17,216 \\
\hline Dennis & Thompson & Harvard U & Harvard U & 1968 & 15,675 \\
\hline Peter & Gourevitch & UC San Diego & Harvard U & 1969 & 15,502 \\
\hline
\end{tabular}

have been listed in The Political Science 40o. A full list is available from the authors on request.

\section{DISCUSSION}

Our main goal was to update the MGF (2007a) dataset with data from more than a decade later and to switch from SSCI data to Google Scholar data, which includes more complete information about book citations. Our data reflect important changes in the profession, including the retirement or death of faculty with $\mathrm{PhDs}$ from the 1960 s and earlier. The ranks of those with PhDs from the 1970 s are already greatly diminished, and further reductions are expected as most in that cohort pass age 75 . Cohorts from the 1990 and later currently comprise a significant proportion of the discipline, and will become an increasing proportion of The Political Science 400 along with cohorts from the 1980 s. Another important change is the growing importance of scholars who identify as methodologists. Although they are relatively few in numbers, their visibility (and influence) is greatly disproportionate to their numbers.

Finally, regarding the status of women in the profession, we observe a growing number of female faculty members in younger cohorts-although many are still in the assistant-professor stage.

Masuoka, Grofman, and colleagues used the dataset they collected to conduct additional analyses of the profession
(Fowler, Grofman, and Masuoka 2007; Masuoka, Grofman, and Feld 2007b; 2007c). Their analyses included reviewing $\mathrm{PhD}$ production rates; examining the ranking of departments by the citations and visibility of both their current faculty and those who received PhDs from the department; comparing those rankings to those provided by other sources (e.g., US News and World Reports); and reviewing the network structure of $\mathrm{PhD}$ placements. In future research, we hope to update some of this work.

\section{SUPPLEMENTARY MATERIAL}

To view supplementary material for this article, please visit https://doi.org/10.1017/S1049096518001786

\section{ACKNOWLEDGMENT}

The authors thank Russell Dalton for providing valuable feedback on previous versions of the dataset.

\section{NOTES}

1. Samuels (2013) is an important exception.

2. Of the 1,002 scholars in American politics, 26 belong in the race and ethnicity subfield; however, they are combined here for space concerns. Public policy, public administration, public law, and political psychology also are combined into one group. 


\section{REFEREN CES}

Fowler, James H., Bernard Grofman, and Natalie Masuoka. 2007. "Social Networks in Political Science: Hiring and Placement of PhDs, 1960-2002." PS: Political Science \& Politics 40 (4): 729-39.

Klingemann, Hans-Dieter. 1986. "Ranking the Graduate Departments in the 1980s: Toward Objective Qualitative Indicators.” PS: Political Science $\mathcal{E}$ Politics $19(3): 651-61$.

Klingemann, Hans-Dieter, Bernard Grofman, and Janet Campagna. 1989. "The Political Science 40o: Citations by PhD Cohort and by PhD-Granting Institution." PS: Political Science \& Politics 22 (2): 258-70.
Masuoka, Natalie, Bernard Grofman, and Scott L. Feld. 2007a. "The Political Science 400: A 20-Year Update." PS: Political Science \& Politics 40 (1): 133-45.

Masuoka, Natalie, Bernard Grofman, and Scott L. Feld. 2007b. "The Production and Placement of PhDs: 1902-200o." PS: Political Science \& Politics 40 (2): 361-66.

Masuoka, Natalie, Bernard Grofman, and Scott L. Feld. 2007c. "Ranking Departments: A Comparison of Alternative Approaches.” PS: Political Science \& Politics $40(3): 531-37$

Samuels, David. 2013. "Book Citations Count.” PS: Political Science \& Politics $46(4): 785-90$ 